ANNALES

POLONICI MATHEMATICI

$95.1(2009)$

\title{
Gauge natural prolongation of connections
}

\author{
by Miroslav Doupovec (Brno) and \\ WŁODZimierz M. Mikulski (Kraków)
}

\begin{abstract}
The main result is the classification of all gauge bundle functors $H$ on the category $\mathcal{P B}_{m}(G)$ which admit gauge natural operators transforming principal connections on $P \rightarrow M$ into general connections on $H P \rightarrow M$. We also describe all gauge natural operators of this type. Similar problems are solved for the prolongation of principal connections to $H P \rightarrow P$. A special attention is paid to linear connections.
\end{abstract}

Introduction. It is well known that various kinds of prolongations of smooth manifolds and geometric structures on them play an important role in many directions of differential geometry and its applications in mathematical physics. The aim of this paper is to study prolongation of principal connections, which has motivation in quantum mechanics, higher order dynamics, field theories and gauge theories of mathematical physics (see [3], [6], [13], [19]). Roughly speaking, by prolongation of connections we understand geometric constructions transforming a given connection on a manifold into a connection on some prolongation of this manifold. We point out that the theory of prolongation of geometric structures has its origins in the works of C. Ehresmann [4], [5]. Significant progress in that research started after the clarification of the role of jet calculus and natural operations in differential geometry [12]. Since that time these problems have been intensively studied by many authors (see e.g. [1], [7]-[12]).

Let $G$ be a finite-dimensional Lie group and denote by $\mathcal{P} \mathcal{B}_{m}(G)$ the category of principal $G$-bundles with $m$-dimensional bases and their local principal $G$-bundle isomorphisms over the identity isomorphism of $G$. It has been pointed out recently that prolongation of principal connections can be expressed in terms of gauge natural operators (see Section 1 below). The present paper is mainly devoted to the following problems:

2000 Mathematics Subject Classification: 58A05, 58A20.

Key words and phrases: general connection, principal connection, gauge bundle functor.

The first author was supported by the project MSM 0021630518. 
Problem 1. Classify all gauge bundle functors $H$ on $\mathcal{P} \mathcal{B}_{m}(G)$ which admit $\mathcal{P} \mathcal{B}_{m}(G)$-gauge natural operators transforming principal connections on $P \rightarrow M$ into general connections on $H P \rightarrow M$.

Problem 2. Classify all gauge bundle functors $H$ on $\mathcal{P} \mathcal{B}_{m}(G)$ such that for every principal bundle $P \rightarrow M$ there is a natural projection $H P \rightarrow P$ which admits $\mathcal{P} \mathcal{B}_{m}(G)$-gauge natural operators transforming principal connections on $P \rightarrow M$ into general connections on $H P \rightarrow P$.

We remark that we have solved similar problems for general connections on an arbitrary fibered manifold $Y \rightarrow M$ (see [2] and Remark 2 below). Moreover, the second author [14] has studied existence of prolongation of connections from $Y \rightarrow M$ to $H Y \rightarrow H M$, where $H$ is a vector bundle functor. One can also introduce prolongation of connections by means of some linear connection on the base manifold (see [9], [12], [16] and [20]). On the other hand, gauge natural operators from Problems 1 and 2 do not need any auxiliary linear connection on $M$.

The structure of the paper is as follows. In Section 1 we recall some basic notions and results we need. In Section 2 we introduce a gauge bundle functor $\widetilde{F}$ which admits $\mathcal{P} \mathcal{B}_{m}(G)$-gauge natural operators transforming principal connections on $P \rightarrow M$ into general connections on $\widetilde{F} P \rightarrow M$. Section 3 is devoted to the solution of Problem 1. In particular, we prove

TheOREM 1*. All gauge bundle functors $H$ which admit gauge natural operators as in Problem 1 are of the form $H P=P[N]$ for some left $G$ space $N$, where $P[N]$ is the fiber bundle associated to $P$ with the fiber $N$.

Moreover, in Theorem 1 below we also describe all such gauge natural operators. In Section 4 we study prolongation of linear connections. Finally, in Section 5 we describe the solution of Problem 2. In what follows we use the terminology and notation from the book [12]. All manifolds and maps are assumed to be infinitely differentiable.

1. The foundations. It has been clarified recently that it is useful to formulate the theory of connections from the point of view of jets, natural bundles and natural operators [12]. This approach leads to the following definition, which generalizes the classical concept of connection.

Definition 1. A general connection on a fibered manifold $p: Y \rightarrow M$ is a smooth section $\Gamma: Y \rightarrow J^{1} Y$ of the first jet prolongation $J^{1} Y$.

Such a $\Gamma$ can be equivalently interpreted as the lifting map (denoted by the same symbol)

$$
\Gamma: Y \times_{M} T M \rightarrow T Y .
$$

Clearly, if $E \rightarrow M$ is a vector bundle, then so also is $J^{1} E$. A connection 
$\Gamma: E \rightarrow J^{1} E$ is called linear if $\Gamma$ is a linear morphism. In the case $E=T M$ of the tangent bundle we obtain the concept of a classical linear connection on $M$. Finally, let $P \rightarrow M$ be a principal $G$-bundle, so that we have the canonical right action $r: J^{1} P \times G \rightarrow J^{1} P$. Then any $r$-invariant connection $\Gamma: P \rightarrow J^{1} P$ is called principal. In what follows we distinguish between general and principal connections.

We denote by $\mathcal{V}$ the category of vector spaces and their linear maps, by $\mathcal{V}_{n}$ the subcategory of $n$-dimensional vector spaces and their isomorphisms, by $\mathcal{M f}$ the category of smooth manifolds and all smooth maps, by $\mathcal{M} f_{m} \subset \mathcal{M} f$ the subcategory of $m$-dimensional manifolds and their local diffeomorphisms, by $\mathcal{F M}$ the category of fibered manifolds and their fiber respecting mappings, by $\mathcal{F} \mathcal{M}_{m, n}$ the subcategory of fibered manifolds with $m$-dimensional bases and $n$-dimensional fibers and their local fibered diffeomorphisms, by $\mathcal{V B}$ the category of vector bundles, by $\mathcal{V} \mathcal{B}_{m, n}$ the subcategory of vector bundles with $m$-dimensional bases and $n$-dimensional fibers, and by $\mathcal{G}$ the category of principal $G$-spaces and their action respecting diffeomorphisms. We recall that a principal $G$-space is a manifold $S$ with a right action of $G$ which is free and transitive. Thus any object $S$ of $\mathcal{G}$ is isomorphic to $G$ with the usual right action of $G$.

Denote by $B: \mathcal{F M} \rightarrow \mathcal{M} f$ the base functor. We recall that a gauge bundle functor on $\mathcal{P B}_{m}(G)$ is a covariant functor $H: \mathcal{P B} \mathcal{B}_{m}(G) \rightarrow \mathcal{F} \mathcal{M}$ such that

(a) every $\mathcal{P B}_{m}(G)$-object $\pi: P \rightarrow B P$ is transformed into a fibered manifold $q_{P}: H P \rightarrow B P$ over $B P$,

(b) every $\mathcal{P} \mathcal{B}_{m}(G)$-morphism $f: P \rightarrow \bar{P}$ is transformed into a fibered morphism $H f: H P \rightarrow H \bar{P}$ over $B f$

(c) for every open subset $U \subset B P$ the inclusion $i: \pi^{-1}(U) \rightarrow P$ is transformed into the inclusion $H i: q_{P}^{-1}(U) \rightarrow H P$.

An example of gauge bundle functor is a $\operatorname{Con}_{G}: \mathcal{P} \mathcal{B}_{m}(G) \rightarrow \mathcal{F M}$, which associates to any principal bundle $P \rightarrow M$ the fiber bundle $\operatorname{Con}_{G}(P) \rightarrow M$ of principal connections on $P \rightarrow M$. The general concept of gauge natural operators can be found in [3] or [12]. In particular, a $\mathcal{P} \mathcal{B}_{m}(G)$-gauge natural operator $D$ transforming principal connections $\Gamma$ on $P \rightarrow M$ into general connections $D(\Gamma)$ on $H P \rightarrow M$ is a system of $\mathcal{P} \mathcal{B}_{m}(G)$-invariant regular operators

$$
D_{P}: \operatorname{Con}_{G}(P) \rightarrow \operatorname{Con}(H P)
$$

for any $\mathcal{P B}_{m}(G)$-object $P \rightarrow M$, where $\operatorname{Con}_{G}(P)$ is the set of all principal connections on $P \rightarrow M$ (i.e. sections of $\operatorname{Con}_{G}(P) \rightarrow M$ ) and $\operatorname{Con}(H P)$ is the set of all general connections on $H P \rightarrow M$. The invariance means that if $\Gamma \in \operatorname{Con}_{G}(P)$ and $\Gamma_{1} \in \operatorname{Con}_{G}\left(P_{1}\right)$ are $f$-related by a $\mathcal{P} \mathcal{B}_{m}(G)$-map $f: P \rightarrow P_{1}$ covering $\underline{f}: M \rightarrow M_{1}$, then $D_{P}(\Gamma)$ and $D_{P_{1}}\left(\Gamma_{1}\right)$ are $H f$-related. 
The regularity means that $D_{P}$ transforms smoothly parametrized families of connections into smoothly parametrized families of connections. Quite similarly one can define $\mathcal{P} \mathcal{B}_{m}(G)$-gauge natural operators $D$ transforming principal connections $\Gamma$ on $P \rightarrow M$ into general connections $D(\Gamma)$ on $H P \rightarrow P$.

One of the most important gauge bundle functors on $\mathcal{P} \mathcal{B}_{m}(G)$ is the $r$ th principal prolongation $W^{r} P$ of a principal bundle $P \rightarrow M$, which is defined as the space of all $r$-jets $j_{(0, \mathrm{e})}^{r} \varphi$ of local trivializations $\varphi: \mathbb{R}^{m} \times G \rightarrow P$, where e $\in G$ is the unit. By [12], $W^{r} P \rightarrow M$ is a principal bundle with the structure group $W_{m}^{r} G:=J_{(0, \mathrm{e})}^{r}\left(\mathbb{R}^{m} \times G, \mathbb{R}^{m} \times G\right)_{(0,-)}$, which can be expressed as the semidirect product $W_{m}^{r} G=G_{m}^{r} \rtimes T_{m}^{r} G$ of $G_{m}^{r}=\operatorname{inv} J_{0}^{r}\left(\mathbb{R}^{m}, \mathbb{R}^{m}\right)_{0}$ and $T_{m}^{r} G=J_{0}^{r}\left(\mathbb{R}^{m}, G\right)$ with respect to the canonical action of $G_{m}^{r}$ on $T_{m}^{r} G$ given by the composition of jets. Moreover, we have

$$
W^{r} P=P^{r} M \times_{M} J^{r} P
$$

where $P^{r} M$ is the $r$ th order frame bundle. We also remark that $W^{r} P$ plays a fundamental role in the theory of gauge bundle functors. Indeed, let $H$ be any gauge bundle functor on $\mathcal{P} \mathcal{B}_{m}(G)$ and denote by $S:=H_{0}\left(\mathbb{R}^{m} \times G\right)$ the fiber over 0 . Then $H$ is of the form

$$
H P=W^{r} P[S],
$$

which is the fiber bundle associated to $W^{r} P$ with the fiber $S$ (see e.g. [12]). In this case $r$ is called the order of $H$. If we replace holonomic jets by nonholonomic or semiholonomic ones, we can define the nonholonomic or semiholonomic principal prolongation $\widetilde{W}^{r} P$ or $\bar{W}^{r} P$, respectively, and the corresponding structure groups $\widetilde{W}_{m}^{r} G$ and $\bar{W}_{m}^{r} G$. Clearly, $\widetilde{W}^{r} P$ can also be defined by iteration, $\widetilde{W}^{r} P=W^{1}\left(\widetilde{W}^{r-1} P\right)$. Taking into account Problem 1 above, there is a question whether one can construct a connection on $W^{r} P \rightarrow$ $M$ from a principal connection on $P \rightarrow M$. Up till now, the only known result of this type is

Proposition 1 ([10]). For $G=G_{m}^{1}$ there is no $\mathcal{P B}_{m}(G)$-gauge natural operator transforming principal connections on $P \rightarrow M$ into connections on $W^{1} P \rightarrow M$.

2. $F$-extension of principal connections. Let $F: \mathcal{G} \rightarrow \mathcal{M} f$ be a regular functor. The regularity means that $F$ transforms smoothly parametrized families of $\mathcal{G}$-maps into smoothly parametrized families of maps. Such a functor can be extended to a gauge bundle functor $\widetilde{F}: \mathcal{P} \mathcal{B}_{m}(G) \rightarrow \mathcal{F} \mathcal{M}$ in the following way:

$$
\widetilde{F} P=\bigcup_{x \in M} F\left(P_{x}\right), \quad \widetilde{F} f=\bigcup_{x \in M} F\left(f_{x}\right)
$$


Consider a principal connection $\Gamma$ on $P \rightarrow M$. Given a vector field $X$ on $M$, its $\Gamma$-lift is a $G$-invariant vector field $\Gamma X: P \rightarrow T P$ on $P$. The flow of $\Gamma X$ is formed by $\mathcal{P B}_{m}(G)$-maps. Since $\widetilde{F}$ is of order zero, the flow prolongation

$$
\widetilde{\mathcal{F}}(\Gamma X)=\frac{\partial}{\partial t_{0}} \widetilde{F}(\exp (t \Gamma X)): \widetilde{F} P \rightarrow T \widetilde{F} P
$$

can be interpreted as a map

$$
\widetilde{\mathcal{F}} \Gamma: \widetilde{F} P \times_{M} T M \rightarrow T \widetilde{F} P .
$$

By linearity, $\widetilde{\mathcal{F}} \Gamma$ is the lifting map of a general connection (denoted by the same symbol) on $\widetilde{F} P \rightarrow M$.

Definition 2. The connection $\widetilde{\mathcal{F}} \Gamma$ is called the $F$-extension of $\Gamma$.

Let $F_{1}, F_{2}: \mathcal{G} \rightarrow \mathcal{M} f$ be regular functors and $K: F_{1} \rightarrow F_{2}$ be a natural transformation. This means that for any $S \in \mathcal{G}$ we have a map $K_{S}: F_{1} S \rightarrow$ $F_{2} S$ such that $K_{S_{2}} \circ F_{1} f=F_{2} f \circ K_{S_{1}}$ for any $\mathcal{G}$-map $f: S_{1} \rightarrow S_{2}$. This natural transformation can be fiberwise extended to a natural transformation $\widetilde{K}: \widetilde{F}_{1} \rightarrow \widetilde{F}_{2}$, where $\widetilde{F}_{1}, \widetilde{F}_{2}: \mathcal{P} \mathcal{B}_{m}(G) \rightarrow \mathcal{F} \mathcal{M}$ are defined by (1). Actually for any $P \rightarrow M$ from $\mathcal{P B}_{m}(G)$ we have

$$
\widetilde{K}_{P}=\bigcup_{x \in M} K_{P_{x}}: \widetilde{F}_{1} P \rightarrow \widetilde{F}_{2} P .
$$

From the canonical character (with respect to $F$ ) of the construction of $\widetilde{\mathcal{F}} \Gamma$ we have

Proposition 2. Let $K: F_{1} \rightarrow F_{2}$ be as above. Given a principal connection $\Gamma$ on $P \rightarrow M$, the general connections $\widetilde{\mathcal{F}}_{1} \Gamma$ and $\widetilde{\mathcal{F}}_{2} \Gamma$ are $\widetilde{K}$-related.

The main result of this section is

Proposition 3. Let $F: \mathcal{G} \rightarrow \mathcal{M f}$ be a regular functor. The $F$-extension $\widetilde{\mathcal{F}} \Gamma$ is the unique $\mathcal{P} \mathcal{B}_{m}(G)$-gauge natural operator $\mathcal{D}$ transforming principal connections $\Gamma$ on $P \rightarrow M$ into general connections $\mathcal{D}(\Gamma)$ on $\widetilde{F} P \rightarrow M$.

To prove Proposition 3 we need some preparations. Let $\mathcal{D}$ be the operator in question and $\Gamma$ be a principal connection on $P \rightarrow M$. Define a map

$$
\Delta(\Gamma):=\mathcal{D}(\Gamma)-\widetilde{\mathcal{F}} \Gamma: \widetilde{F} P \times_{M} T M \rightarrow V(\widetilde{F} P \rightarrow M) .
$$

It remains to show that $\Delta(\Gamma)=0$. Let $\mathbb{R}^{m} \times G$ be the trivial principal $G$-bundle over $\mathbb{R}^{m}$. Let $x^{1}, \ldots, x^{m}$ be the usual coordinates on $\mathbb{R}^{m}$ and $Y_{1}, \ldots, Y_{n}$ a basis of right $G$-invariant vector fields on $G$. Let $y \in$ $\left(\widetilde{F}\left(\mathbb{R}^{m} \times G\right)\right)_{0}=F G$ and $v \in T_{0} \mathbb{R}^{m}$. By the invariance of $\Delta$ with respect to principal bundle trivialization, it suffices to prove

$$
\Delta(\Gamma)(y, v)=0 \in T_{y} F G
$$

for any principal connection $\Gamma$ on $\mathbb{R}^{m} \times G$. We first prove two lemmas. 
Lemma 1. Suppose that

$$
\Delta\left(\sum_{i=1}^{m} d x^{i} \otimes \frac{\partial}{\partial x^{i}}+\sum_{i=1}^{m} \sum_{j=1}^{n} \sum_{|\alpha| \leq K} \Gamma_{i \alpha}^{j} x^{\alpha} d x^{i} \otimes Y_{j}\right)(y, v)=0
$$

for any $K \in \mathbb{N}$ and any $\Gamma_{i \alpha}^{j} \in \mathbb{R}$ for any m-tuple $\alpha$ with $|\alpha| \leq K$ and $i=1, \ldots, m$ and $j=1, \ldots, n$. Then we have (3).

Proof. This follows from the nonlinear Peetre theorem (see Corollary 19.8 in $[12])$.

Lemma 2. Suppose that

$$
\Delta\left(\sum_{i=1}^{m} d x^{i} \otimes \frac{\partial}{\partial x^{i}}+d x^{i_{o}} \otimes Y_{j_{o}}\right)(y, v)=0
$$

for any $i_{o}=1, \ldots, m$ and $j_{o}=1, \ldots, n$. Then we have (3).

Proof. Using the invariance of $\Delta$ with respect to the $\mathcal{P B}_{m}(G)$-maps $t \operatorname{id}_{\mathbb{R}^{m}} \times \operatorname{id}_{G}$ for $t>0$ we get the homogeneity condition

$$
\begin{aligned}
\Delta\left(\sum_{i=1}^{m} d x^{i}\right. & \left.\otimes \frac{\partial}{\partial x^{i}}+\sum_{i=1}^{m} \sum_{j=1}^{n} \sum_{|\alpha| \leq K} \Gamma_{i \alpha}^{j} t^{|\alpha|+1} x^{\alpha} d x^{i} \otimes Y_{j}\right)(y, v) \\
& =t \Delta\left(\sum_{i=1}^{m} d x^{i} \otimes \frac{\partial}{\partial x^{i}}+\sum_{i=1}^{m} \sum_{j=1}^{n} \sum_{|\alpha| \leq K} \Gamma_{i \alpha}^{j} x^{\alpha} d x^{i} \otimes Y_{j}\right)(y, v) .
\end{aligned}
$$

By the homogeneous function theorem (Theorem 24.1 from [12]), this type of homogeneity implies that

$$
\Delta\left(\sum_{i=1}^{m} d x^{i} \otimes \frac{\partial}{\partial x^{i}}+\sum_{i=1}^{m} \sum_{j=1}^{n} \sum_{|\alpha| \leq K} \Gamma_{i \alpha}^{j} x^{\alpha} d x^{i} \otimes Y_{j}\right)(y, v)
$$

depends linearly on $\Gamma_{i(0)}^{j}$ and is independent of $\Gamma_{i \alpha}^{j}$ for $|\alpha|>0$. So the assumption of the lemma implies the assumption of Lemma 1, which completes the proof.

Proof of Proposition 3. Clearly, from the proof of Lemma 2 it follows that

$$
\Delta\left(\sum_{i=1}^{m} d x^{i} \otimes \frac{\partial}{\partial x^{i}}\right)(y, v)=0
$$

Let $\psi_{t}$ be the flow of the $G$-invariant vector field $Y_{j_{o}}$. Define a map $K$ : $\mathbb{R}^{m} \times G \rightarrow \mathbb{R}^{m} \times G$ by

$$
K(x, \xi)=\left(x, \psi_{x^{i_{o}}}(\xi)\right) .
$$

It is locally a $\mathcal{P} \mathcal{B}_{m}(G)$-map sending $\partial / \partial x^{i_{o}}$ into $\partial / \partial x^{i_{o}}+Y_{j_{o}}$ and preserving $\partial / \partial x^{i}$ for $i=1, \ldots, m$ with $i \neq i_{o}$ and $d x^{i}$ for $i=1, \ldots, m$. As 
$K(0, \xi)=(0, \xi)$, we have $\widetilde{F} K=$ id over zero. So $K$ preserves also $(y, v)$. Using invariance of $\Delta$ with respect to $K$ we see that (4) implies the assumption of Lemma 2, which completes the proof of (3).

3. Solution of Problem 1. Consider a left $G$-space $N$ and denote by $F^{[N]}: \mathcal{P} \mathcal{B}_{m}(G) \rightarrow \mathcal{F} \mathcal{M}$ the gauge bundle functor defined by

$$
F^{[N]} P=P[N]=P \times_{G} N, \quad F^{[N]} f=f \times_{G} \operatorname{id}_{N},
$$

where $P[N]$ is the fiber bundle associated to $P$ with the fiber $N$.

A principal connection $\Gamma$ on $P \rightarrow M$ induces a general connection $\Gamma[N]$ on $P[N] \rightarrow M$ in the following way. Every element $u \in P[N]$ is an equivalence class $u=\{p, n\}, p \in P, n \in N$. If $\Gamma(p)=j_{x}^{1} \sigma$, where $\sigma: M \rightarrow P$ is a local section, then we can define

$$
\Gamma[N](u)=j_{x}^{1}\{\sigma, n\} .
$$

As $\Gamma$ is right-invariant, the induced connection $\Gamma[N]$ is well defined. Clearly, the correspondence $\Gamma \mapsto \Gamma[N]$ is a $\mathcal{P B}_{m}(G)$-gauge natural operator.

TheOREM 1. Let $H: \mathcal{P B}_{m}(G) \rightarrow \mathcal{F M}$ be a gauge bundle functor. $A \mathcal{P B}_{m}(G)$-gauge natural operator $\mathcal{D}$ transforming principal connections $\Gamma$ on $P \rightarrow M$ into general connections $\mathcal{D}(\Gamma)$ on $H P \rightarrow M$ exists if and only if $H$ is isomorphic to $F^{[N]}$, where $F^{[N]} P=P[N]$ for some left $G$-space $N$. For $H=F^{[N]}$ such an operator $\mathcal{D}$ is unique and we have $\mathcal{D}(\Gamma)=\Gamma[N]$.

The rest of this section will be devoted to the proof of Theorem 1. Clearly, a right $G$-space $S \in \mathcal{G}$ can be considered as a trivial principal bundle $S \rightarrow$ pt, where pt is a one-element manifold. Given an arbitrary left $G$-space $N$, we have a regular functor $F^{N}: \mathcal{G} \rightarrow \mathcal{M} f$ defined by

$$
F^{N} S=S \times_{G} N, \quad F^{N} f=f \times_{G} \operatorname{id}_{N},
$$

where $S \times{ }_{G} N$ is the trivial fiber bundle associated to $S$.

Lemma 3. Every regular functor $F: \mathcal{G} \rightarrow \mathcal{M f}$ is isomorphic to $F^{N}$ for some left $G$-space $N$.

Proof. Write $N:=F(G)$ with the induced left action of $G$ given by $g \cdot n=F\left(L_{g}\right)(n)$. Then the isomorphism $\Phi_{S}: F^{N} S \rightarrow F S$ is defined by $\Phi_{S}(\{s, n\})=F(\widetilde{s})(n)$, where $\widetilde{s}: G \rightarrow S$ is the unique $\mathcal{G}$-map such that $\widetilde{s}\left(\mathrm{e}_{G}\right)=s$.

For $F=F^{N}: \mathcal{G} \rightarrow \mathcal{M} f$ the corresponding gauge bundle functor $\widetilde{F}$ : $\mathcal{P} \mathcal{B}_{m}(G) \rightarrow \mathcal{F} \mathcal{M}$ from Section 2 will be denoted by $\widetilde{F}^{N}$. From (1) we have $\widetilde{F}^{N}=F^{[N]}$, so that Theorem 1 follows directly from

Proposition 4. Let $H$ be a gauge bundle functor on $\mathcal{P B}_{m}(G)$. There exists a $\mathcal{P} \mathcal{B}_{m}(G)$-gauge natural operator $\mathcal{D}$ transforming principal connections $\Gamma$ on $P \rightarrow M$ into general connections $\mathcal{D}(\Gamma)$ on $H P \rightarrow M$ if and only 
if $H$ is isomorphic to $\widetilde{F}$ for some regular functor $F: \mathcal{G} \underset{\widetilde{\mathcal{F}}}{\rightarrow} \mathcal{M} f$. If $H=\widetilde{F}$, then such an operator $\mathcal{D}$ is unique and we have $\mathcal{D}(\Gamma)=\widetilde{\mathcal{F}} \Gamma$.

Now we are going to prove Proposition 4 . Suppose that $\mathcal{D}$ is an operator as in the statement and define a natural bundle $H^{1}$ over $m$-manifolds by

$$
H^{1} M=H(M \times G), H^{1} \psi=H\left(\psi \times \operatorname{id}_{G}\right) .
$$

LEMMA 4. $H^{1}$ is of order zero.

Proof. Define an $\mathcal{M} f_{m}$-natural operator $A: T \rightsquigarrow T H^{1}$ transforming vector fields $X$ on $m$-manifolds $M$ into vector fields $A(X)$ on $H^{1} M$ by

$$
A(X)=X^{\mathcal{D}\left(\Gamma_{M}\right)},
$$

the horizontal lifting of $X$ with respect to $\mathcal{D}\left(\Gamma_{M}\right)$, where $\Gamma_{M}$ is the trivial principal connection on the trivial $G$-bundle $M \times G \rightarrow M$. Clearly, $A$ is of order zero. On the other hand, $A(X)$ is projectable over $X$. Then we have

$$
A=\mathcal{H}^{1}+\mathcal{V}
$$

for some vertical type natural operator $\mathcal{V}: T \rightsquigarrow T H^{1}$, where $\mathcal{H}^{1}$ is the flow operator. Suppose that $H^{1}$ has (minimal) order $r \geq 1$. Then $\mathcal{H}^{1}$ is of minimal order $r$. By Lemma 1 in [15], $\mathcal{V}$ is of order $r-1$. Then $A$ is not of order zero, which is a contradiction.

Lemma 5. Let $f: P_{1} \rightarrow P_{2}$ be a $\mathcal{P} \mathcal{B}_{m}(G)$-map covering $f: M_{1} \rightarrow M_{2}$, and let $x \in M_{1}$. Then $(H f)_{x}$ depends (only) on $f_{x}:\left(P_{1}\right)_{x} \rightarrow\left(P_{2}\right)_{f(x)}$.

Proof. We may assume

$$
P_{1}=P_{2}=\mathbb{R}^{m} \times G, \quad x=0, \quad f_{0}=\mathrm{id} .
$$

Let $v \in H_{0}\left(\mathbb{R}^{m} \times G\right)$. It remains to prove $H f(v)=v$. In general, $f: \mathbb{R}^{m} \times G \rightarrow$ $\mathbb{R}^{m} \times G$ is of the form $f(x, \xi)=(f(x), \tilde{f}(x, \xi))$. Because $H^{1}$ is of order zero, replacing $f$ by $\left(f^{-1} \times \operatorname{id}_{G}\right) \circ f$ we can assume that

$$
f(x, \xi)=(x, \tilde{f}(x, \xi)) .
$$

Further, as $H^{1}$ is of order zero, we have

$$
H f(v)=H^{1}\left(\frac{1}{t} \operatorname{id}_{\mathbb{R}^{m}}\right) \circ H f \circ H^{1}\left(t \operatorname{id}_{\mathbb{R}^{m}}\right)(v)=H\left(p, \tilde{f} \circ\left(t \operatorname{id}_{\mathbb{R}^{m}} \times \operatorname{id}_{G}\right)\right)(v),
$$

where $p: \mathbb{R}^{m} \times G \rightarrow \mathbb{R}^{m}$ is the projection. Using regularity of $H$ and letting $t \rightarrow 0$ we get

$$
H f(v)=H\left(\operatorname{id}_{\mathbb{R}^{m}} \times \operatorname{id}_{G}\right)(v)=v,
$$

which ends the proof.

Proof of Proposition 4. Define a functor $F: \mathcal{G} \rightarrow \mathcal{M f}$ by

$$
F S=H_{0}\left(\mathbb{R}^{m} \times S\right), \quad F g=H_{0}\left(\mathrm{id}_{\mathbb{R}^{m}} \times g\right) .
$$


Further, define a fibered map $I_{P}: H P \rightarrow \widetilde{F} P$ covering $\operatorname{id}_{M}$ by

$$
I_{P}(v)=H f(v) \in H_{0}\left(\mathbb{R}^{m} \times P_{x_{o}}\right)=F\left(P_{x_{o}}\right)=(\widetilde{F} P)_{x_{o}},
$$

where $v \in(H P)_{x_{o}}, x_{o} \in M$ and $f: P \rightarrow \mathbb{R}^{m} \times P_{x_{o}}$ is a (local) $\mathcal{P} \mathcal{B}_{m}(G)$-map such that $\underline{f}\left(x_{o}\right)=0$ and $f_{x_{o}}=$ id. By Lemma 5 , the definition of $I_{P}(v)$ is independent of the choice of $f$. The inverse map is $J_{P}: \widetilde{F} P \rightarrow H P$ defined by

$$
J_{P}(w)=H f^{-1}(w), \quad w \in H_{0}\left(\mathbb{R}^{m} \times P_{x_{o}}\right)=(\widetilde{F} P)_{x_{o}}, \quad x_{o} \in M,
$$

where $f$ is as above. Regularity of $H$ implies the smoothness of $I_{P}$ and $J_{P}$, so that $I_{P}$ is a diffeomorphism. From the functoriality of $H$ it follows directly that $I: H \rightarrow \widetilde{F}$ is a $\mathcal{P} \mathcal{B}_{m}(G)$-gauge natural transformation. Finally, the second part of Proposition 4 is exactly Proposition 3.

Corollary 1. Let $H$ be any of the functors $J^{r}, W^{r}, \widetilde{W}^{r}$ and $\bar{W}^{r}$. There is no $\mathcal{P B}_{m}(G)$-gauge natural operator transforming principal connections on $P \rightarrow M$ into general connections on $\mathrm{HP} \rightarrow M$.

We can see that Corollary 1 generalizes Proposition 1. For the identity functor $F=$ id $: \mathcal{G} \rightarrow \mathcal{M} f_{m}, \widetilde{F}$ is the identity functor on $\mathcal{P} \mathcal{B}_{m}(G)$. So we have

COROLlary 2. Any $\mathcal{P} \mathcal{B}_{m}(G)$-gauge natural operator $\mathcal{D}$ transforming principal connections $\Gamma$ on $P \rightarrow M$ into general connections on $P \rightarrow M$ is of the form $\mathcal{D}(\Gamma)=\Gamma$.

Definition 3. Let $F: \mathcal{M} f_{m} \rightarrow \mathcal{F M}$ be a natural bundle. A general connection on $F M \rightarrow M$ is said to be canonical if it is invariant with respect to local diffeomorphisms of $\mathcal{M} f_{m}$.

For example, if $F$ is a trivial natural bundle $F M=M \times S \rightarrow M$ for some manifold $S$, then the trivial connection on $F M \rightarrow M$ is canonical. Clearly, for the one-element group $G=\{e\}$, gauge bundle functors on $\mathcal{P} \mathcal{B}_{m}(G)$ are exactly natural bundles on $\mathcal{M} f_{m}$. Theorem 1 implies

Proposition 5. Let $F: \mathcal{M} f_{m} \rightarrow \mathcal{F M}$ be a natural bundle. A canonical general connection on $F M \rightarrow M$ exists if and only if $F$ is isomorphic to the trivial natural bundle $F M=M \times S \rightarrow M$ for some manifold $S$. For $M \times S \rightarrow M$ such a connection is unique and is equal to the trivial one.

For the tangent functor $T: \mathcal{M} f_{m} \rightarrow \mathcal{V B}$ we have

COROLlary 3. There is no canonical classical linear connection on $M$.

4. Prolongation of linear connections. One verifies directly that for $G=\operatorname{GL}(n)$ the categories $\mathcal{V}_{n}$ and $\mathcal{G}$ are equivalent. Clearly, this equivalence is given by $V \mapsto \mathrm{L}(V)$, where $\mathrm{L}(V)$ is the principal $\mathrm{GL}(n)$-space of all bases of a vector space $V$. Quite analogously we find that the categories $\mathcal{V} \mathcal{B}_{m, n}$ 
and $\mathcal{P B}_{m}(\mathrm{GL}(n))$ are equivalent (see also Chapter 54.1 in [12]). So linear connections on a vector bundle $E \rightarrow M$ correspond bijectively to principal $\mathrm{GL}(n)$-connections on $\mathrm{L}(E) \rightarrow M$, where $\mathrm{L}(E)=\bigcup_{x \in M} \mathrm{~L}\left(E_{x}\right)$. From Proposition 4 we have

Proposition 6. Let $H: \mathcal{V B}_{m, n} \rightarrow \mathcal{F M}$ be a gauge bundle functor. $A \mathcal{V} B_{m, n}$-gauge natural operator $\mathcal{D}$ transforming linear connections $\Gamma$ on a vector bundle $E \rightarrow M$ into general connections $\mathcal{D}(\Gamma)$ on $H E \rightarrow M$ exists if and only if $H$ is isomorphic to $\widetilde{F}$ for some regular functor $F: \mathcal{V}_{n} \rightarrow \mathcal{M} f$. If $H=\widetilde{F}$, then such an operator $\mathcal{D}$ is unique and we have $\mathcal{D}(\Gamma)=\widetilde{\mathcal{F}} \Gamma$.

Corollary 4. Let $\Gamma$ be a linear connection on a vector bundle $E \rightarrow M$ and denote by $\Gamma^{*}$ the dual connection on $E^{*} \rightarrow M$. Then the correspondence $\Gamma \mapsto \bigotimes^{k} \Gamma \otimes \bigotimes^{l} \Gamma^{*}$ is the only $\mathcal{V} \mathcal{B}_{m, n}$-gauge natural operator transforming linear connections on $E \rightarrow M$ into linear connections on $\bigotimes^{k} E \otimes \bigotimes^{l} E^{*} \rightarrow M$.

Clearly, the conclusion of Proposition 2 is also true for the natural transformation $K: F_{1} \rightarrow F_{2}$ of arbitrary regular functors $F_{1}, F_{2}: \mathcal{V}_{n} \rightarrow \mathcal{V}$. In particular, Proposition 2 yields

Corollary 5. Let $F_{1} E=\bigotimes^{k} E \otimes \bigotimes^{l} E^{*}, F_{2} E=\bigotimes^{k-1} E \otimes \bigotimes^{l-1} E^{*}$ and let $K: F_{1} \rightarrow F_{2}$ be a contraction. Then $\widetilde{\mathcal{F}}_{1} \Gamma$ and $\widetilde{\mathcal{F}}_{2} \Gamma$ are $\widetilde{K}$-related.

Roughly speaking, this means that gauge natural operators $\Gamma \mapsto \bigotimes^{k} \Gamma \otimes$ $\bigotimes^{l} \Gamma^{*}$ commute with contractions. We point out that this is well-known in the case of classical linear connections $\Gamma=\nabla$.

COROLlaRY 6. There is no $\mathcal{V} \mathcal{B}_{m, n^{-}}$gauge natural operator transforming linear connections on a vector bundle $E \rightarrow M$ into linear connections on the $r$-jet prolongation $J^{r} E \rightarrow M$.

REMARK 1. The second author has recently solved some existence problems on prolongation of classical linear connections. In particular, he characterized all natural bundles $F$ which admit natural operators transforming classical linear connections on $M$ into classical linear connections on $F M$ (see [17]).

5. Solution of Problem 2. Given a manifold $Q$ we have the trivial gauge bundle functor $E^{Q}$ on $\mathcal{P B}_{m}(G)$ defined by

$$
E^{Q} P=P \times Q, \quad E^{Q} f=f \times \operatorname{id}_{Q} .
$$

From now on $E^{Q} P \rightarrow P$ means the usual projection onto the first factor.

ExAmple 1. Let $A: Q \times \mathcal{L}(G) \rightarrow T Q$ be a vector bundle homomorphism covering $\operatorname{id}_{Q}$, where $\mathcal{L}(G)=T_{e} G$ is the Lie algebra of $G$. Let $\Gamma$ be a principal connection on $P \rightarrow M$ and let $\omega_{\Gamma}: T P \rightarrow \mathcal{L}(G)$ be its classical connection 
form. We can define a general connection

$$
\mathcal{V}_{A}^{Q} \Gamma: E^{Q} P \times_{P} T P \rightarrow T E^{Q} P
$$

on $E^{Q} P \rightarrow P$ by

$$
\left.\mathcal{V}_{A}^{Q} \Gamma((p, q), v)\right)=\left(v, A\left(q, \omega_{\Gamma}(v)\right)\right) \in T_{p} P \times T_{q} Q=T_{(p, q)} E^{Q} P,
$$

where $(p, q) \in E^{Q} P$ and $v \in T_{p} P$.

Proposition 7. All $\mathcal{P B}_{m}(G)$-gauge natural operators transforming principal connections $\Gamma$ on $P \rightarrow M$ into general connections on $E^{Q} P \rightarrow P$ are the operators $\mathcal{V}_{A}^{Q}$ from Example 1.

Proof. Let $\mathcal{D}$ be an operator as in the statement. Define a vector bundle map $A: Q \times \mathcal{L}(G) \rightarrow T Q$ covering $\operatorname{id}_{Q}$ by

$$
(\xi, A(q, \xi))=\mathcal{D}\left(\Gamma_{\mathbb{R}^{m}}\right)(((0, e), q), \xi) \in T_{e} G \times T_{q} Q
$$

where $q \in Q, \xi \in \mathcal{L}(G)=T_{e} G=V_{(0, e)}\left(\mathbb{R}^{m} \times G\right)$ and $\Gamma_{\mathbb{R}^{m}}$ is the trivial principal connection on the trivial principal $G$-bundle $\mathbb{R}^{m} \times G$ and where we use the identification $V_{((0, e), q)}\left(E^{Q}\left(\mathbb{R}^{m} \times G\right) \rightarrow \mathbb{R}^{m}\right)=T_{e} G \times T_{q} Q$. Now we prove that $\mathcal{D}=\mathcal{V}_{A}^{Q}$. Indeed, let $\Gamma$ be a principal connection on a $\mathcal{P} \mathcal{B}_{m}(G)$ object $P \rightarrow M$. Define a map

$$
\Delta(\Gamma):=\mathcal{D}(\Gamma)-\mathcal{V}_{A}^{Q}: E^{Q} P \times T P \rightarrow V\left(E^{Q} P \rightarrow P\right) .
$$

It remains to show that $\Delta(\Gamma)=0$. Let $q \in Q$ and $v \in T_{(0, e)}\left(\mathbb{R}^{m} \times G\right)$. Using the invariance of $\Delta$ with respect to $G$-bundle trivialization we see that it suffices to prove

$$
\Delta(\Gamma)(((0, e), q), v)=0 \in T_{q} Q
$$

for any principal connection $\Gamma$ on $\mathbb{R}^{m} \times G \rightarrow \mathbb{R}^{m}$. Assume first that $v \in$ $V_{(0, e)}\left(\mathbb{R}^{m} \times G\right)=T_{e} G$. By the invariance of $\Delta$ with respect to $\mathcal{P} \mathcal{B}_{m}(G)$-maps $t \operatorname{id}_{\mathbb{R}^{m}} \times \operatorname{id}_{G}, t \neq 0$, and letting $t \rightarrow 0$, because of the definitions of $A$ and $\Delta$ we get

$$
\Delta(\Gamma)(((0, e), q), v)=\Delta\left(\Gamma_{\mathbb{R}^{m}}\right)(((0, e), q), v)=0 \in T_{q} Q .
$$

Further, assume $v \in H_{(0, e)}^{\Gamma}$. Define a $\mathcal{P} \mathcal{B}_{m}(G)$-gauge natural operator $\mathcal{D}_{1}$ transforming principal connections $\Gamma$ on $P \rightarrow M$ into general connections $\mathcal{D}_{1}(\Gamma)$ on $E^{Q} P \rightarrow M$ by

$$
\mathcal{D}_{1}(\Gamma)((p, q), w)=\mathcal{D}(\Gamma)((p, q), \Gamma(p, w)),
$$

where $(p, q) \in\left(E^{Q} P\right)_{x}, w \in T_{x} M, x \in M$. By Proposition 4 we have

$$
\mathcal{D}_{1}(\Gamma)((p, q), w)=\Gamma(p, w) \in T_{p} P=T_{p} P \times\left\{0_{q}\right\} \subset T_{(p, q)} E^{Q} P .
$$

Then we have (7) and the proof is complete.

TheOREM 2. Let $H: \mathcal{P B}_{m}(G) \rightarrow \mathcal{F M}$ be a gauge bundle functor such that there is a natural projection $\pi: H P \rightarrow P$ for any $\mathcal{P} \mathcal{B}_{m}(G)$-object $P \rightarrow M$. 
There exists a $\mathcal{P} \mathcal{B}_{m}(G)$-gauge natural operator $\mathcal{D}$ transforming principal connections $\Gamma$ on $P \rightarrow M$ into general connections $\mathcal{D}(\Gamma)$ on $\pi: H P \rightarrow P$ if and only if $H \cong E^{Q}$ for some $Q$ via a $\mathcal{P B}_{m}(G)$-gauge natural isomorphism of bundles $\pi: H P \rightarrow P$ and $E^{Q} P \rightarrow P$ over $\operatorname{id}_{P}$. If $H=E^{Q}$ with the projection $E^{Q} P \rightarrow P$, then all such operators are $\mathcal{D}=\mathcal{V}_{A}^{Q}$ from Example 1 .

Proof. Suppose that there is an operator $\mathcal{D}$ as above. Given a principal connection $\Gamma$ on $P \rightarrow M$, we can define a general connection $\widetilde{\mathcal{D}}(\Gamma)$ on $H P \rightarrow M$ by

$$
\widetilde{\mathcal{D}}(\Gamma)(y, w)=\mathcal{D}(\Gamma)(y, \Gamma(p, w))
$$

where $y \in(H P)_{p}, p \in P_{x}, w \in T_{x} M, x \in M$. So we have a $\mathcal{P} \mathcal{B}_{m}(G)$ gauge natural operator $\widetilde{\mathcal{D}}$ transforming principal connections $\Gamma$ on $P \rightarrow M$ into general connections on $H P \rightarrow M$. This means that the assertion of Lemma 5 is also true for the gauge bundle functor $H$ from Theorem 2 . Define a manifold $Q$ by

$$
Q=\left(H\left(\mathbb{R}^{m} \times G\right)\right)_{(0, e)} .
$$

We prove that $\pi: H P \rightarrow P$ is isomorphic to $E^{Q} P \rightarrow P$. Define a fibered map $I_{P}: H P \rightarrow E^{Q} P$ covering the identity of $P$ by

$$
I_{P}(v)=\left(p_{o}, H f(v)\right) \in\left\{p_{o}\right\} \times H_{(0, e)}\left(\mathbb{R}^{m} \times G\right)=\left\{p_{o}\right\} \times Q=\left(H^{Q} P\right)_{p_{o}},
$$

where $v \in(H P)_{p_{o}}, p_{o} \in P$ and $f: P \rightarrow \mathbb{R}^{m} \times G$ is a (local) $\mathcal{P} \mathcal{B}_{m}(G)$-map such that $f\left(p_{o}\right)=(0, e)$. By Lemma 5 , the definition of $I_{P}(v)$ is independent of the choice of $f$ (for, if $\widetilde{f}: \mathbb{R}^{m} \times G \rightarrow \mathbb{R}^{m} \times G$ is a $\mathcal{P} \mathcal{B}_{m}(G)$-map such that $\widetilde{f}(0, e)=(0, e)$, then $\left.\widetilde{f}_{0}=\mathrm{id}\right)$. The inverse map is $J_{P}: E^{Q} P \rightarrow H P$ defined by

$$
J_{P}(w)=H f^{-1}(w), \quad w \in\left(E^{Q} P\right)_{p_{o}}, \quad p_{o} \in P,
$$

where $f$ is as above. Regularity of $H$ implies the smoothness of $I_{P}$ and $J_{P}$, so that $I_{P}$ is a diffeomorphism. Finally, from the functoriality of $H$ it follows directly that $I: H \rightarrow E^{Q}$ is a $\mathcal{P} \mathcal{B}_{m}(G)$-gauge natural transformation.

Corollary 7. Let $H$ be any of the functors $J^{r}, W^{r}, \widetilde{W}^{r}$ and $\bar{W}^{r}$. There is no $\mathcal{P B}_{m}(G)$-gauge natural operator transforming principal connections on $P \rightarrow M$ into general connections on $H P \rightarrow P$.

Clearly, the classical linear connection on $P$ is the linear connection on $T P \rightarrow P$. Theorem 2 implies (see also [18])

COROLlary 8. There is no $\mathcal{P B}_{m}(G)$-gauge natural operator transforming principal connections on $P \rightarrow M$ into classical linear connections on $P$.

On the other hand, I. Kolář [11] has determined all $\mathcal{P} \mathcal{B}_{m}(G)$-gauge natural operators transforming a principal connection on $P \rightarrow M$ and a symmetric linear connection on the base manifold $M$ into a classical linear connection on P. Moreover, J. Gancarzewicz and I. Kolář [7] have solved a similar 
problem in the case of a vector bundle $P \rightarrow M$. By Corollary 8 , the use of an auxiliary linear connection on the base manifold in such geometric constructions is unavoidable.

REMARK 2. Let $Q$ be a manifold and $Y \rightarrow M$ a fibered manifold. In [2] we have proved that for a bundle functor $H$ on $\mathcal{F} \mathcal{M}_{m, n}$ there is a construction of the general connection $\mathcal{D}(\Gamma)$ on $H Y \rightarrow Y$ from a general connection $\Gamma$ on $Y \rightarrow M$ if and only if $H Y=Y \times Q$ for some manifold $Q$. Moreover, if $H Y=Y \times Q$, then such an operator $\mathcal{D}(\Gamma)$ is unique and is equal to the trivial connection on $Y \times Q \rightarrow Y$. We can see that the existence part of Theorem 2 is similar to the above mentioned result from [2]. On the other hand, the additional part of Theorem 2 is diametrically different. Indeed, in the case of general connections on fibered manifolds we have only one natural operator $\mathcal{D}$, while the gauge natural operators $\mathcal{V}_{A}^{Q}$ from Theorem 2 are parametrized by vector bundle homomorphisms $A$.

\section{References}

[1] J. Dębecki, Affine liftings of torsion-free connections to Weil bundles, Colloq. Math. 114 (2009), 1-8.

[2] M. Doupovec and W. M. Mikulski, On the existence of prolongation of connections, Czechoslovak Math. J. 56 (2006), 1323-1334.

[3] D. Eck, Gauge-natural bundles and generalized gauge theories, Mem. Amer. Math. Soc. 33 (1981), no. 247.

[4] C. Ehresmann, Les prolongements d'un espace fibré différentiable, C. R. Acad. Sci. Paris 240 (1955), 1755-1757.

[5] —, Sur les connexions d'ordre supérieur, in: Atti del V. Congr. Un. Mat. Italiana, 1955, Cremonese, Roma, 1956, 344-346.

[6] L. Fatibene and M. Francaviglia, Natural and Gauge Natural Formalism for Classical Field Theories, Kluwer, 2003.

[7] J. Gancarzewicz and I. Kolár̆, Some gauge-natural operators on linear connections, Monatsh. Math. 111 (1991), 23-33.

[8] J. Janyška, On natural operations with linear connections, Czechoslovak Math. J. 35 (110) (1985), 106-115.

[9] I. Kolář, On some operations with connections, Math. Nachr. 69 (1975), 297-306.

[10] - Some natural operators in differential geometry, in: Differential Geometry and its Applications (Brno, 1986), Reidel, Dordrecht, 1987, 91-110.

[11] —, Some gauge-natural operators on connections, in: Differential Geometry, Colloq. Math. Soc. János Bolyai 56, János Bolyai Math. Soc., Budapest, and Elsevier, Amsterdam, 1992, 435-445.

[12] I. Kolář, P. W. Michor and J. Slovák, Natural Operations in Differential Geometry, Springer, 1993.

[13] L. Mangiarotti and M. Modugno, Fibered spaces, jet spaces and connections for field theories, in: Proc. Int. Meeting on Geometry and Physics (Florence, 1982), Pitagora, Bologna, 1983, 135-165.

[14] W. M. Mikulski, Non-existence of some canonical constructions on connections, Comment. Math. Univ. Carolin. 44 (2003), 691-695. 
[15] W. M. Mikulski, Non-existence of natural operators transforming connections on $Y \rightarrow M$ into connections on $F Y \rightarrow Y$, Arch. Math. (Brno) 41 (2005), 1-4.

[16] - A construction of a connection on $G Y \rightarrow Y$ from a connection on $Y \rightarrow M$ by means of classical linear connections on $M$ and $Y$, Comment. Math. Univ. Carolin. 46 (2005), 759-770.

[17] —, The natural bundles admitting natural lifting of linear connections, Demonstratio Math. 39 (2006), 223-232.

[18] - Negative answers to some questions about constructions on connections, ibid. 39 (2006), 685-689.

[19] M. Palese and E. Winterroth, Gauge-natural field theories and Noether theorems: canonical covariant conserved currents, Rend. Circ. Mat. Palermo (2) Suppl. 79 (2006), 161-174.

[20] F. W. Pohl, Connexions in differential geometry of higher order, Trans. Amer. Math. Soc. 125 (1966), 310-325.

Department of Mathematics

Brno University of Technology

FSI VUT Brno, Technická 2

61669 Brno, Czech Republic

E-mail: doupovec@fme.vutbr.cz
Institute of Mathematics Jagiellonian University

Łojasiewicza 6

30-348 Kraków, Poland E-mail: mikulski@im.uj.edu.pl

Received 28.4.2008

and in final form 19.9.2008 